\title{
SUPERSOFT X-RAY SOURCE RX J0019.8+2156
}

\author{
KATSURA MATSUMOTO \\ Department of Astronomy, Kyoto University, Japan . \\ E-mail: katsura@kusastro.kyoto-u.ac.jp \\ AND \\ MASAMITSU OKUGAMI AND JUN FUKUE \\ Astronomical Institute, Osaka Kyoiku University, Japan
}

\section{Irradiation by White Dwarf}

RX J0019.8+2156 (RX J0019) is one of supersoft X-ray sources (SSXSs) which were rarely found in the Galaxy, and the brightest one upto now (Beuermann et al. 1995). Although the binary systems of SSXSs are still not perfectly revealed, if the model with nuclear burning white dwarf (van den Heuvel et al. 1992) is adopted, it is supposed that most of radiative energy are generated on the surface of the white dwarf. The radiation strongly irradiates the accretion disk and companion, and the reprocessing has an influence upon an observed flux of SSXSs in lower frequencies (Popham, DiStefano 1996).

\section{Modeling of Irradiation Effect and Light Curve}

We assumed the nuclear burning on the white dwarf to be isotoropic, and the accretion disk obeys the standard model. Then, the heating rate on the disk by irradiation (e.g., Fukue 1992) can be estimated for RX J0019. The main parameters are mass and inclination angle; where the mass of the white dwarf is set to be $1.2 \mathrm{M}_{\odot}$, and $L_{\mathrm{wd}}=4 \times 10^{36} \mathrm{erg} \mathrm{s}^{-1}$. The distance to the object is fixed to $2 \mathrm{kpc}$ for this calculation. In order to compare the theoretical energy distributions with observed fluxes, we used the UV (Gänsicke et al. 1996) and optical results (Matsumoto 1996).

With the standard accretion disk model, the optical and UV fluxes of expected luminosities are less than the observed value by factor of about $5-15$. We next considered the accretion disk and companion with the irradi- 


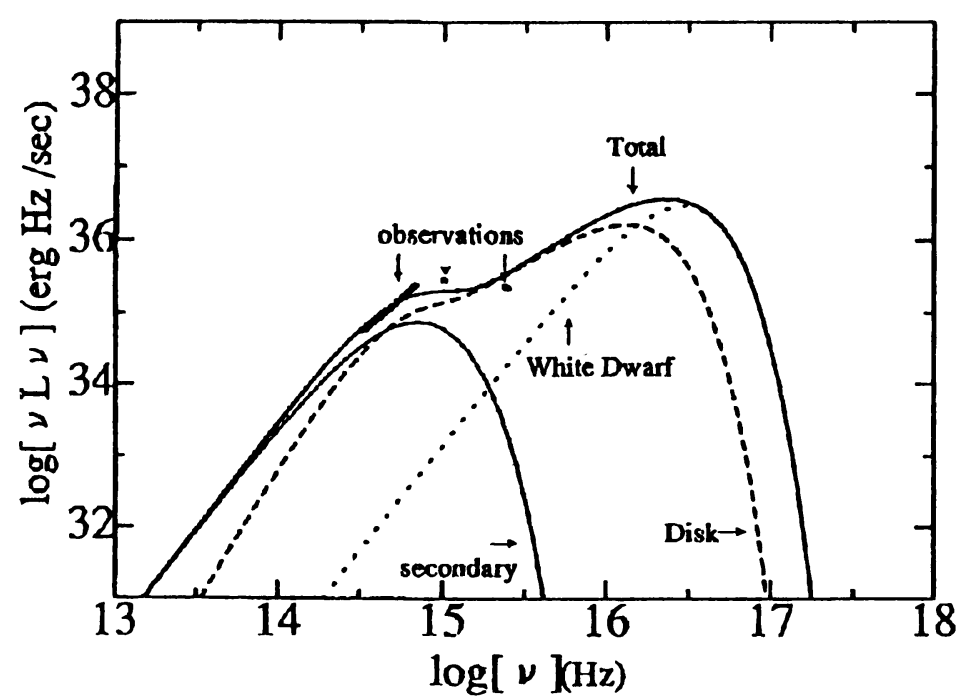

Figure 1. The expected energy distribution of RX J0019 with irradiation effects.

ation effect (figure 1). The energy distribution exhibits clearly increasing of UV and optical fluxes by factor of about 3-5 compared with the standard disk model. The expected energy distributions for the parameters reproduce close concord with the observed results for relatively lower inclination angle. The flux of the companion is accordingly smaller, and the importance of the irradiated accretion disk enlarges especially in the lower frequency. As a result, observations in UV or optical for the object provide reprocessed radiation of X-ray generated on the white dwarf.

Then we calculated optical light curves using this model. Inclination angles of $i=70-80^{\circ}$ can reproduce the shape well like the observations around the photometric minimum. It is supposed that the lower inclination system may be rejected, and the higher inclination system is favorite for observations in $B$ and $V$ band. While, a parameter of $i \sim 50^{\circ}$ has relatively good reproducing for the amplitude. As for the variations of color index, the case of $i=70-80^{\circ}$ seems to be too high to reproduce the observations.

\section{References}

Beuermann, K. et al. 1995, Astron. Astrophys., 294, L1

Fukue, J. 1992, Publ. Astron. Soc. Japan, 44, 669

Gänsicke, B. T., Beuermann, K. and deMartino, D. 1996, Supersoft X-Ray Sources, Springer-Verlag, pp 107

Matsumoto, K. 1996, Publ. Astron. Soc. Japan, 48, 827

Popham, R. and DiStefano, R. 1996, Supersoft X-Ray Sources, Springer-Verlag, pp 65

van den Heuvel, E.P.J., Bhattacharya, D., Nomoto, K. and Rappaport, S. A. 1992, Astron. Astrophys., 262, 97 